\title{
AXL is a marker for epithelial-mesenchymal transition in esophageal squamous cell carcinoma
}

\author{
GUOAN ZHANG $^{1 *}$, XIA KONG ${ }^{2 *}$, MENG WANG $^{3}$, HONGLI ZHAO $^{4}$, \\ SHA HAN ${ }^{5}$, RONGHANG HU ${ }^{6}$, JIAN HUANG ${ }^{7}$ and WEN CUI ${ }^{1}$ \\ ${ }^{1}$ Cancer Pathology Research Institute, Jining Medical University, Jining, Shandong 272067; Departments of ${ }^{2}$ Pharmacy \\ and ${ }^{3}$ Oncology, Shandong Jining No. 1 People's Hospital, Jining, Shandong 272011; ${ }^{4}$ Department of Gastroenterology, \\ Shandong Control Center for Digestive Diseases, Jining, Shandong 272033; ${ }^{5}$ Life Science Experimental Center, \\ Jining Medical University, Jining, Shandong 272067; ${ }^{6}$ Department of Thoracic Surgery; ${ }^{7}$ Central Laboratory, \\ Affiliated Hospital of Jining Medical University, Jining, Shandong 272029, P.R. China
}

Received December 17, 2016; Accepted October 20, 2017

DOI: $10.3892 / \mathrm{ol} .2017 .7443$

\begin{abstract}
Esophageal squamous cell carcinoma (ESCC) is a common cancer in China and certain other parts of the world with a dismal prognosis for affected patients. AXL is a member of the TYRO3-AXL-MER family of receptor tyrosine kinases, and has been revealed to be an important mediator of epithelial-mesenchymal transition (EMT) in several types of cancer. However, to the best of our knowledge, its function in EMT in ESCC cells has not yet been examined. The present study employed two independent ESCC mRNA profile datasets and revealed that AXL is associated with several EMT markers. Gene Set Enrichment Analysis indicated that EMT occurs more in ESCC with high AXL expression. Analysis on another dataset demonstrated further that increased expression of AXL in ESCC is associated with increased migratory ability. Collectively, the results of the present study provide evidence that AXL is a marker for EMT in ESCC.
\end{abstract}

\section{Introduction}

AXL is a member of the TYRO3-AXL-MER family of receptor tyrosine kinases, which includes two other members: TYRO3 and MER. AXL is activated by its ligand, growth arrest-specific 6, which in turn triggers several downstream signaling pathways depending on the cell type, primarily phosphoinositide 3-kinase (PI3K)/protein kinase B (AKT) and

Correspondence to: Dr Wen Cui, Cancer Pathology Research Institute, Jining Medical University, 16 Beihu Road, Jining, Shandong 272067, P.R. China

E-mail: cuiwenmdd@163.com

*Contributed equally

Key words: esophageal squamous cell carcinoma, AXL, epithelial-mesenchymal transition mitogen-activated protein kinase/extracellular-signal-regulated kinase, nuclear factor- $\kappa \mathrm{B}(\mathrm{NF}-\kappa \mathrm{B})$ and signal transducer and activator of transcription 3 (STAT3) signaling pathways. It exerts a wide array of functions including cell survival, proliferation, migration and adhesion (1). AXL has also been implicated in the pathophysiology of a number of types of cancer, including breast, gastric, prostate, ovarian and lung (2-4). AXL expression promotes cancer cell survival, angiogenesis, metastasis and drug resistance $(3,5)$.

AXL is significantly associated with epithelial-mesenchymal transition (EMT) in several tumors. RNA sequencing data of 643 cancer cell lines demonstrated that AXL expression is markedly associated with a mesenchymal phenotype (6). AXL expression is increased in mesenchymal cells compared with epithelial cells in non-small cell lung cancer (NSCLC) cell lines (7). Furthermore, AXL downregulation in A549 and H460 mesenchymal cells leads to enhanced expression of epithelial cadherin (E-cadherin) and decreased expression of vimentin and neural cadherin (N-cadherin), which are features of mesenchymal-epithelial transition $(7,8)$, suggesting that AXL is involved in maintaining EMT. AXL has also been identified to regulate EMT in other types of cancer, including breast $(9,10)$, prostate $(11)$, ovarian $(12)$ and pancreatic $(13,14)$.

China is among the countries at highest risk of esophageal squamous cell carcinoma (ESCC) (15). ESCC is the fourth most common cancer in China, accounting for $\sim 13 \%$ of all cancer cases in 2015 (16). ESCC is frequently associated with a high risk of recurrence and high mortality rate and the 5-year survival rate is $<20 \%$ (17).

The function of AXL in ESCC has been demonstrated only recently (18-20). AXL gene (19) and protein $(19,20)$ expression were upregulated in ESCC cells compared with normal adjacent cells, and were identified to be associated with tumor progression, increased risk of mortality and distant metastasis (20). AXL was also identified to be consistently overexpressed in ESCC cell lines (19). Knockdown of AXL expression inhibited cell proliferation, survival, migration and invasion in vitro and in vivo, and those effects were mediated by the AKT/NF- $\mathrm{B}$ and AKT/glycogen synthase kinase- $3 \beta$ signaling pathways (19). Overexpression of AXL 
also mediated resistance to the PI3K $\alpha$ inhibitor BYL719 by activating signaling pathways in ESCC cells (18).

However, the function of AXL in EMT in ESCC cells is not well-documented. To the best of our knowledge, the present study provides the first evidence that $\mathrm{AXL}$ is a marker for the mesenchymal phenotype in ESCC using online mRNA profile data.

\section{Materials and methods}

Gene expression data. GSE47404 (21), GSE23400 (22) and GSE21293 (23) gene expression data were obtained from the Gene Expression Omnibus database (GEO; www.ncbi.nlm. nih.gov/geo). Further information on the sample size and the microarray platforms used for the creation of these datasets is presented in Table I. Processed expression data and information of corresponding platforms were downloaded and used further. Expression values (mRNA $\log _{2}$ intensity) for each gene were examined manually and used for further study.

Gene Set Enrichment Analysis (GSEA). GSEA is a computational software that determines whether predefined set of genes exhibit statistically significant concordant differences between two biological states (http://software.broadinstitute. org/gsea/index.jsp) (24). AXL expression was determined on the basis of GSE47404 and GSE23400 datasets. If two probe sets correspond to AXL, AXL expression is calculated as the average of the value of the two probe sets. Following determination of the mean expression of AXL, the samples were designated AXL-high ESCC (samples with an above-average AXL value) or AXL-low ESCC (samples with a below-average AXL value). AXL-low samples served as the control samples. This collection of gene sets summarized and represented specific well-defined biological states or processes, and was generated by a computational methodology based on identifying gene set overlaps and retaining genes that display coordinate expression across several datasets (25). All the parameters were set to their default values. A false discovery rate (FDR) value $<0.25$ was considered to indicate significant enrichment. The procedure followed for the GSEA is presented in Fig. 1.

Association of AXL expression and cancer cell invasive ability in GSE21293. GSE21293 displayed the mRNA profiles of 34 genetically engineered human esophageal cells with different invasive abilities (23). To determine whether AXL is associated with the invasive ability of ESCC cells, AXL expression in the samples was examined and the mean expression value was calculated, and samples were divided into two groups according to their AXL expression value as aforementioned: AXL-high and AXL-low. AXL-low samples acted as the control samples. The samples were also divided into two groups according to their invasive ability: Invasive and non-invasive. Fisher's exact test was used to examine their association. Furthermore, AXL expression in the invasive and non-invasive groups was also compared using Mann-Whitney U test.

Statistical analysis. Data were analyzed using SPSS (version 13.0; SPSS, Inc., Chicago, IL, USA). Spearman's correlation

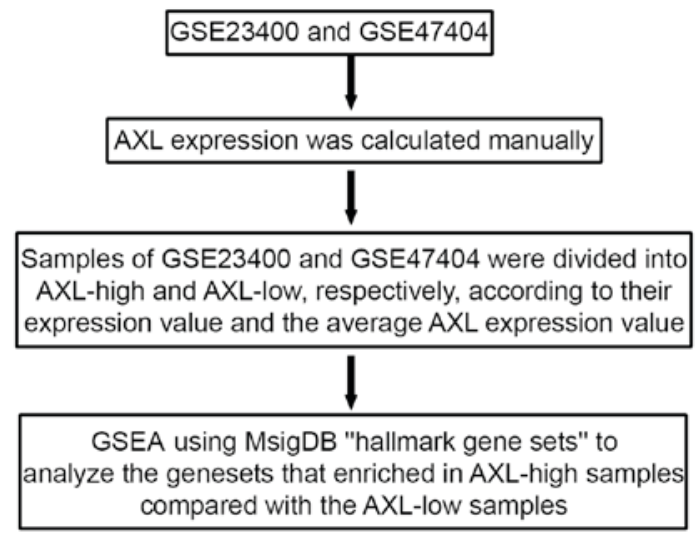

Figure 1. Schematic diagram of the procedure for the GSEA. GSEA, Gene Set Enrichment Analysis; MsigDB, The Molecular Signatures Database.

was used to analyze the association between AXL mRNA and mRNA of mesenchymal markers. Fisher's Exact Test was applied to determine the correlation between AXL expression and invasion ability of genetically engineered esophageal cells. Mann-Whitney $U$ test was used to assess the expression difference of AXL mRNA in invasive and non-invasive esophageal cells. $\mathrm{P}<0.05$ was considered to indicate a statistically significant difference.

\section{Results}

AXL mRNA expression is associated with certain mesenchymal markers. To investigate the function of AXL in EMT, the association of AXL with several EMT markers, including E-cadherin, vimentin, fibronectin, snail family transcriptional repressor 1 (SNAI1), snail family transcriptional repressor 2 (SNAI2), twist family basic helix-loop-helix transcription factor 1 (TWIST1), zinc finger E-box-binding homeobox 1 (ZEB1) and N-cadherin, was examined (Fig. 2). It was revealed that AXL mRNA expression in GSE47404 and GSE23400 datasets is associated with several EMT markers, including vimentin $\left(\mathrm{P}=1.00 \times 10^{-6}\right.$ for GSE47404 and $1.00 \times 10^{-6}$ for GSE23400; Fig. 2B and J), fibronectin ( $\mathrm{P}=2.22 \times 10^{-4}$ for

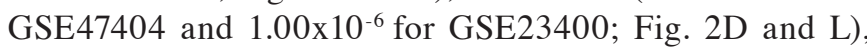
TWIST1 $\left(\mathrm{P}=0.01\right.$ for GSE47404 and $1.90 \times 10^{-4}$ for GSE23400; Fig. 2G and O) and ZEB1 ( $\mathrm{P}=0.04$ for GSE47404 and $1.00 \times 10^{-6}$ for GSE23400; Fig. $2 \mathrm{H}$ and P). However, no association between AXL expression and E-cadherin (Fig. 2A and I) or SNAI2 (Fig. 2F and N) was identified in either dataset. A significant association was identified between AXL and SNAI1 in GSE47404 (P=2.03 $\times 10^{-3}$; Fig. 2E), but not in GSE23400 ( $\mathrm{P}=0.67$; Fig. 2M). Additionally, the association between AXL and N-cadherin was significant in GSE23400 ( $\mathrm{P}=6.59 \times 10^{-3}$; Fig. 2K), but not in GSE47404 ( $\mathrm{P}=0.74$; Fig. 2C). These preliminary results suggest that AXL may be involved in EMT in ESCC.

EMT gene set is enriched in the ESCC group with high AXL expression. To further determine the function of AXL in EMT in ESCC, GSEA was performed to examine whether the AXL-high and the AXL-low expression groups display a different EMT state in ESCC. The GSEA of the present 
Table I. Gene expression data.

Dataset accession no.

Description

Platform

GSE47404 mRNA profiles of 71 primary tumor tissues from patients with ESCC from Japan

GSE23400

GSE21293
mRNA profiles of 51 pairs of primary tumor tissues from patients with ESCC and adjacent normal tissues from China (only mRNA expression profiles from patients with ESCC were used in this study) mRNA profiles of invasive and non-invasive genetically engineered human esophageal cells with hTERT and p53 mutations and ERFG overexpression
Whole Human Genome 4X44K Agilent G4112F microarray Human Genome U133 Set microarray

Human Genome U133Plus 2.0 microarray

ESCC, esophageal squamous cell carcinoma; hTERT, human telomerase reverse transcriptase; EGFR, epidermal growth factor receptor.
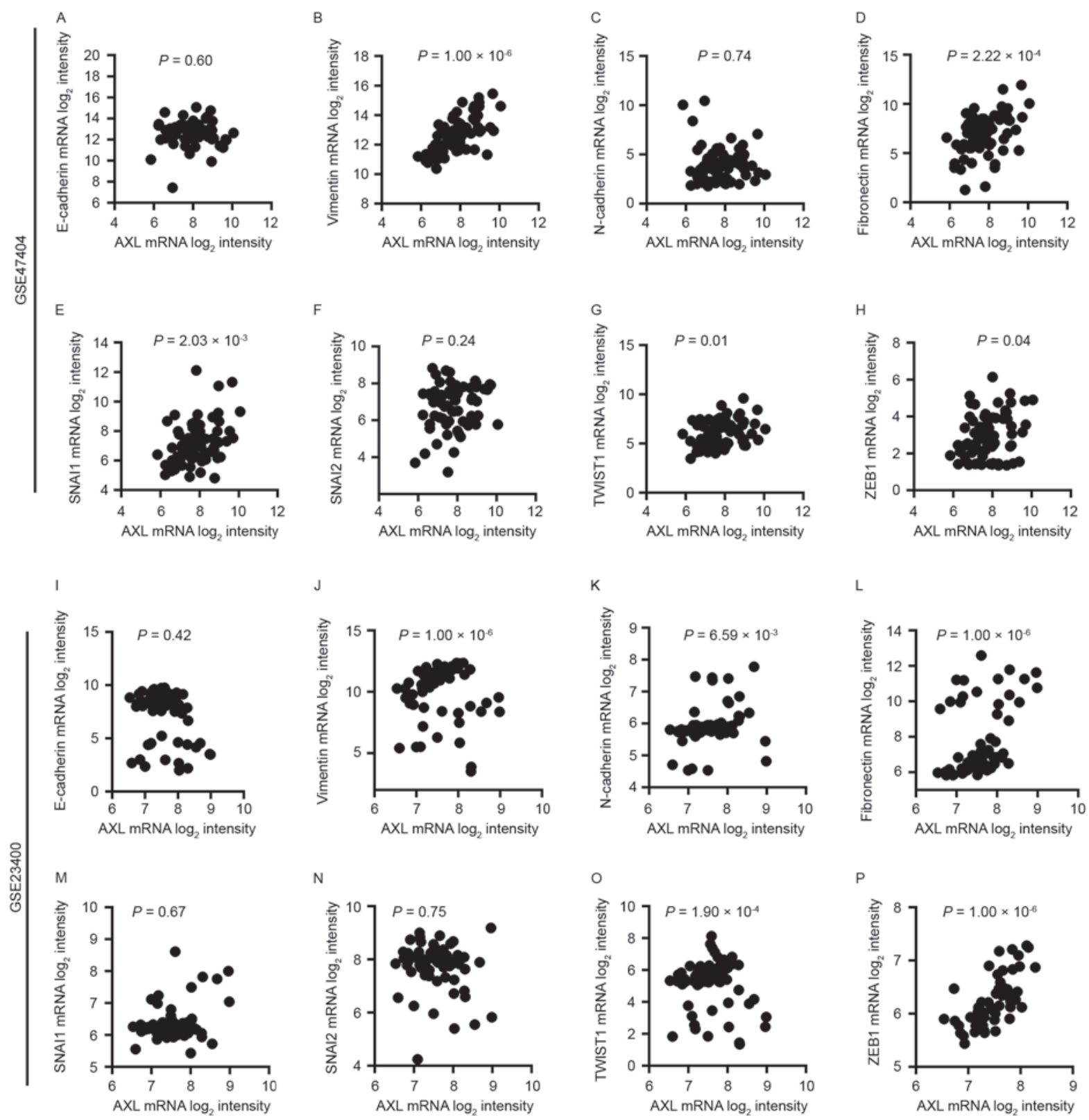

Figure 2. Association between the expression of AXL and (A) E-cadherin, (B) vimentin, (C) N-cadherin, (D) fibronectin, (E) SNAI1, (F) SNAI2, (G) TWIST1 and (H) ZEB1 in the GSE47404 dataset. Association between the expression of AXL and (I) E-cadherin, (J) vimentin, (K) N-cadherin, (L) fibronectin, (M) SNAI1, (N) SNAI2, (O) TWIST1 and (P) ZEB1 in the GSE23400 dataset. Results were assessed by Spearman's correlation. E-cadherin, epithelial cadherin; N-cadherin, neural cadherin; SNAI, snail family transcriptional repressor; TWIST1, twist family basic helix-loop-helix transcription factor 1; ZEB1, zinc finger E-box-binding homeobox 1. 

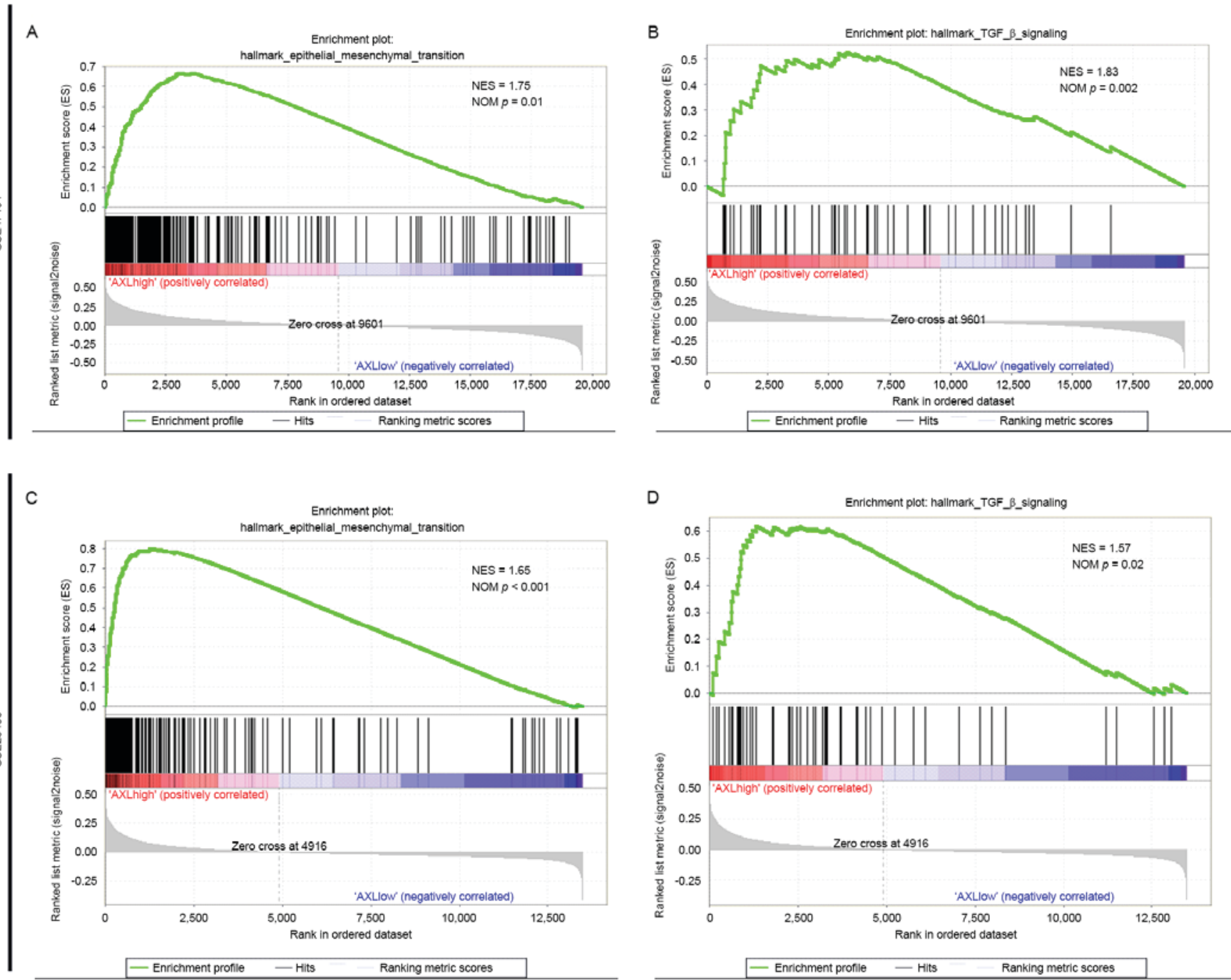

Figure 3. Gene Set Enrichment Analysis demonstrated that the (A) epithelial-mesenchymal transition gene set and the (B) TGF $\beta$ gene set were enriched in the ESCC group with high AXL expression in the GSE47404 dataset. Gene Set Enrichment Analysis demonstrated that the (C) epithelial-mesenchymal transition gene set and the (D) TGF $\beta$ gene set were enriched in the ESCC group with high AXL expression in the GSE23400 dataset. The barcode plot indicates the position of the genes in each gene set. The horizontal bar in graded color from red to blue indicates positive and negative correlation with AXL expression. The vertical axis in the lower plot indicates Ranked List Metric. NES, normalized enrichment score; NOM, nominal; TGF $\beta$, transforming growth factor- $\beta$.

study utilized 'Hallmark gene sets [h.all.v5.2.symbols.gmt (Hallmarks)]' as the gene sets to be analyzed.

The results revealed that in GSE47404, the ESCC group with high AXL expression levels displayed a more marked mesenchymal state compared with the ESCC group with low AXL expression levels (Fig. 3A, and Table II). Furthermore, transforming growth factor- $\beta$ signaling, which has a predominant function in EMT in cancer $(26,27)$, is also enriched in the AXL-high expression group (Fig. 3B and Table II). Analyze on GSE23400 also showed similar pattern (Fig. 3C and D and Table II). STAT3 signaling, which is reported to serve a function in EMT in ESCC (28), is also highly enriched in the AXL-high expression group in the two datasets (Table II).

AXL expression is associated with increased invasive ability of ESCC cells. A prominent feature of EMT is to confer enhanced invasive ability on cancer cells. Therefore, the present study assessed the association between AXL expression and the invasive ability of ESCC on the basis of the GSE21293 dataset (23). This dataset includes normal esophageal cells, which are genetically engineered with human telomerase reverse transcriptase, epidermal growth factor receptor overexpression and p53 mutations, in order to immortalize them and display differential invasion. According to their ability to invade, these cells were divided into invasive and non-invasive groups. As presented in Table III, the AXL-high expression group displayed increased ability of invasion $\left(\mathrm{P}=2.61 \times 10^{-4}\right)$. AXL expression in non-invasive and invasive groups was also compared and it was revealed that the invasive group expressed significantly increased AXL levels $\left(\mathrm{P}=1.95 \times 10^{-4}\right.$; Fig. 4).

\section{Discussion}

EMT is a fundamental process in body development, and in the differentiation of multiple tissues and organs (29). EMT endows cancer cells with invasive properties and with stem-cell like characteristics, prevents drug-induced apoptosis and senescence, and contributes to immunosuppression $(29,30)$. The EMT state defined by the E-cadherin/vimentin ratio has 
Table II. Gene Set Enrichment Analysis on GSE47404 and GSE23400 with hallmark gene sets.

\begin{tabular}{|c|c|c|c|c|}
\hline $\begin{array}{l}\text { Dataset } \\
\text { accession no. }\end{array}$ & Gene set name & NES & $\begin{array}{l}\text { NOM } \\
\text { P-value }\end{array}$ & $\begin{array}{c}\text { FDR } \\
\text { Q-value }\end{array}$ \\
\hline GSE47404 & HALLMARK_ALLOGRAFT_REJECTION & 1.87 & $<0.001$ & 0.15 \\
\hline GSE47404 & HALLMARK_NOTCH_SIGNALING & 1.85 & $<0.001$ & $9.59 \times 10^{-2}$ \\
\hline GSE47404 & HALLMARK_COMPLEMENT & 1.84 & $<0.001$ & $6.73 \times 10^{-2}$ \\
\hline GSE47404 & HALLMARK_COAGULATION & 1.84 & $<0.001$ & $5.04 \times 10^{-2}$ \\
\hline GSE47404 & HALLMARK_TGF_BETA_SIGNALING & 1.83 & $1.95 \times 10^{-3}$ & $4.38 \times 10^{-2}$ \\
\hline GSE47404 & HALLMARK_IL2_STAT5_SIGNALING & 1.82 & $<0.001$ & $3.95 \times 10^{-2}$ \\
\hline GSE47404 & HALLMARK_ANGIOGENESIS & 1.79 & $7.39 \times 10^{-3}$ & $4.98 \times 10^{-2}$ \\
\hline GSE47404 & $\begin{array}{l}\text { HALLMARK_EPITHELIAL_ } \\
\text { MESENCHYMAL_TRANSITION }\end{array}$ & 1.75 & $1.13 \times 10^{-2}$ & $4.67 \times 10^{-2}$ \\
\hline GSE47404 & HALLMARK_IL6_JAK_STAT3_SIGNALING & 1.73 & $5.93 \times 10^{-3}$ & $4.73 \times 10^{-2}$ \\
\hline GSE47404 & $\begin{array}{l}\text { HALLMARK_INTERFERON_ } \\
\text { GAMMA_RESPONSE }\end{array}$ & 1.73 & $1.79 \times 10^{-2}$ & $4.58 \times 10^{-2}$ \\
\hline GSE47404 & HALLMARK_INFLAMMATORY_RESPONSE & 1.71 & $1.38 \times 10^{-2}$ & $4.83 \times 10^{-2}$ \\
\hline GSE47404 & HALLMARK_UV_RESPONSE_DN & 1.64 & $1.24 \times 10^{-2}$ & $7.29 \times 10^{-2}$ \\
\hline GSE47404 & HALLMARK_KRAS_SIGNALING_UP & 1.63 & $5.78 \times 10^{-3}$ & $7.14 \times 10^{-2}$ \\
\hline GSE47404 & HALLMARK_APOPTOSIS & 1.62 & $3.12 \times 10^{-2}$ & $6.76 \times 10^{-2}$ \\
\hline GSE47404 & $\begin{array}{l}\text { HALLMARK_INTERFERON_ } \\
\text { ALPHA_RESPONSE }\end{array}$ & 1.62 & $3.43 \times 10^{-2}$ & $6.54 \times 10^{-2}$ \\
\hline GSE47404 & $\begin{array}{l}\text { HALLMARK_TNFA_SIGNALING_ } \\
\text { VIA_NFKB }\end{array}$ & 1.53 & $8.74 \times 10^{-2}$ & 0.10 \\
\hline GSE47404 & HALLMARK_HYPOXIA & 1.38 & 0.11 & 0.20 \\
\hline GSE23400 & HALLMARK_COAGULATION & 1.71 & $5.86 \times 10^{-3}$ & $7.27 \times 10^{-2}$ \\
\hline GSE23400 & HALLMARK_KRAS_SIGNALING_UP & 1.71 & $<0.001$ & $3.64 \times 10^{-2}$ \\
\hline GSE23400 & HALLMARK_UV_RESPONSE_DN & 1.66 & $1.91 \times 10^{-3}$ & $4.74 \times 10^{-2}$ \\
\hline GSE23400 & $\begin{array}{l}\text { HALLMARK_EPITHELIAL_ } \\
\text { MESENCHYMAL_TRANSITION }\end{array}$ & 1.65 & $<0.001$ & $4.51 \times 10^{-2}$ \\
\hline GSE23400 & HALLMARK_APICAL_JUNCTION & 1.58 & $4.10 \times 10^{-3}$ & $8.88 \times 10^{-2}$ \\
\hline GSE23400 & HALLMARK_TGF_BETA_SIGNALING & 1.57 & $1.74 \times 10^{-2}$ & $7.69 \times 10^{-2}$ \\
\hline GSE23400 & HALLMARK_ANGIOGENESIS & 1.55 & $2.10 \times 10^{-2}$ & $8.26 \times 10^{-2}$ \\
\hline GSE23400 & HALLMARK_MYOGENESIS & 1.54 & $3.97 \times 10^{-2}$ & $7.94 \times 10^{-2}$ \\
\hline GSE23400 & HALLMARK_ALLOGRAFT_REJECTION & 1.54 & $4.45 \times 10^{-2}$ & $7.36 \times 10^{-2}$ \\
\hline GSE23400 & HALLMARK_COMPLEMENT & 1.49 & $3.75 \times 10^{-2}$ & 0.10 \\
\hline GSE23400 & HALLMARK_IL6_JAK_STAT3_SIGNALING & 1.47 & $6.22 \times 10^{-2}$ & 0.11 \\
\hline GSE23400 & HALLMARK_IL2_STAT5_SIGNALING & 1.45 & $<0.001$ & 0.11 \\
\hline GSE23400 & HALLMARK_APOPTOSIS & 1.45 & $9.77 \times 10^{-3}$ & 0.11 \\
\hline GSE23400 & HALLMARK_INFLAMMATORY_RESPONSE & 1.41 & $7.68 \times 10^{-2}$ & 0.13 \\
\hline GSE2340 & $\begin{array}{l}\text { HALLMARK_TNFA_SIGNALING_- } \\
\text { VIA_NFKB }\end{array}$ & 1.36 & 0.01 & 0.17 \\
\hline GSE23400 & $\begin{array}{l}\text { HALLMARK_INTERFERON_ } \\
\text { GAMMA_RESPONSE }\end{array}$ & 1.31 & 0.18 & 0.23 \\
\hline GSE23400 & HALLMARK_PROTEIN_SECRETION & 1.31 & 0.12 & 0.21 \\
\hline
\end{tabular}

NOM P-values indicate differences between AXL-high ESCC group and AXL-low ESCC group for the corresponding gene set. FDR Q-values indicate the estimated probability that the normalized enrichment score represents a false positive finding. FDR, false discovery rate; IL, interleukin; Jak, Janus kinase; NES, normalized enrichment score; NOM, nominal; STAT, signal transducer and activator of transcription; TGF, transforming growth factor; TNF, tumor necrosis factor; UV, ultraviolet.

been demonstrated to be associated with a poor 5-year survival rate of patients with ESCC (31).

AXL has been demonstrated to be an important factor in certain tumors including NSCLC, breast cancer, prostate cancer, myeloid leukemia and ovarian cancer $(2,3,12,32)$. AXL enhances tumor proliferation, promotes EMT and induces drug resistance $(2,3,32)$. Clinical trials for melanoma, NSCLC and acute myeloid leukemia are ongoing to determine the 
Table III. Association between AXL expression and invasive ability of genetically engineered esophageal cells.

Invasive ability

\begin{tabular}{lcccc} 
& Non-invasive & Invasive & Total & P-value \\
\cline { 2 - 5 } AXL-low & 19 & 2 & 21 & 14 \\
AXL-high & 4 & 10 & 12 & $2.61 \times 10^{-4}$ \\
Total & 23 & 12 &
\end{tabular}

Results were assessed using Fisher's exact test.

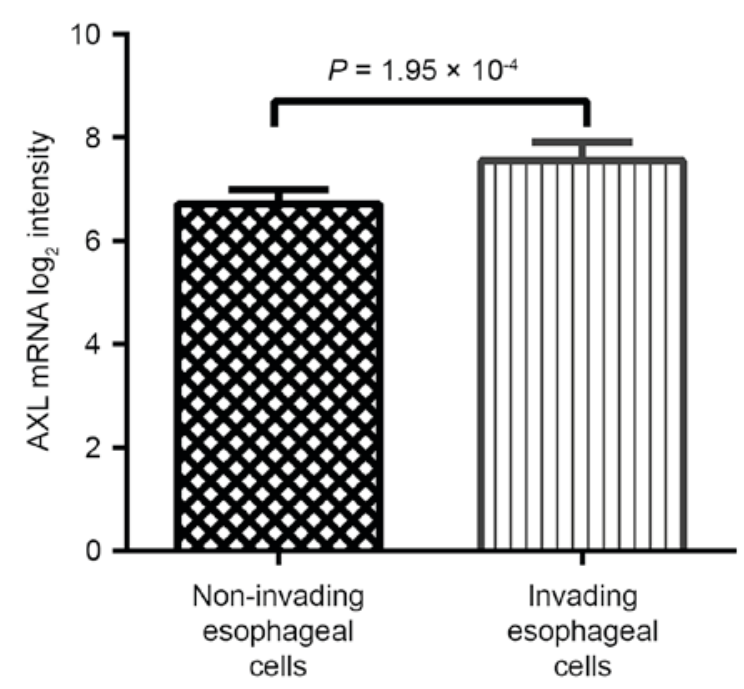

Figure 4. Expression of AXL in non-invasive and invasive esophageal cells. Results were assessed by Mann-Whitney U test.

safety and efficiency of AXL inhibitors (https://clinicaltrials. gov/ct $2 /$ results?cond $=\&$ term $=$ BGB324\&cntry $1=\&$ state $1=\&$ Se $\operatorname{arch}=$ Search).

The function of AXL in ESCC has only recently been identified (18-20). However, its function in EMT in ESCC is even less well-elucidated. The present study provides preliminary evidence from three independent mRNA array studies indicating that AXL is a marker for the mesenchymal state of ESCC. According to the results of the present study, it was revealed that AXL expression is associated with certain EMT markers, including vimentin, fibronectin, TWIST1 and ZEB1. However, AXL was not associated with other EMT markers, including E-cadherin. These results are consistent with those in a study by Wilson et al (6) which demonstrated that AXL mRNA is associated with vimentin mRNA expression in a group of 643 human cancer cell lines. In contrast, Sato et al (33) indicated that AXL expression is associated with E-cadherin, but not vimentin, in lung adenocarcinomas. Additionally, the ESCC group with high AXL expression was enriched with the EMT gene set as revealed by GSEA. Another data cohort revealed that AXL is markedly associated with the invasive ability of genetically engineered esophageal cells. Collectively, the results of the present study suggest that AXL is an EMT marker for ESCC. However, further studies, including in vitro and in vivo cancer models, are required to confirm those results. As mRNA and protein expression are not necessarily associated (34), experiments analyzing AXL protein levels in ESCC are required.

\section{Acknowledgements}

The present study is supported by a Shandong Province Higher Educational Science and Technology Program (grant no. J13LK14), Natural Science Foundation of Shandong province (grant no. 2013ZRC03007), Jining Scientific and Technological Project (grant nos. 2012jnjc02, 2014jnjc17 and 2015-57-71), Youth Foundation of Jining Medical University (grant nos. JY2013KJ035 and JY2015KJ023), The Research Innovation Program for College Graduates of Shandong Province (grant no. SDYY14014) and Projects of Education Planning Office of Shandong Province (grant no. 2013GG043). The authors thank Dr Liyan Guo (Associate Professor of Epidemiology, Jining Medical University) for assistance with statistical analysis.

\section{References}

1. Hafizi S and Dahlbäck B: Signalling and functional diversity within the Axl subfamily of receptor tyrosine kinases. Cytokine Growth Factor Rev 17: 295-304, 2006

2. Paccez JD, Vogelsang M, Parker MI and Zerbini LF: The receptor tyrosine kinase $\mathrm{Axl}$ in cancer: Biological functions and therapeutic implications. Int J Cancer 134: 1024-1033, 2014.

3. Graham DK, DeRyckere D, Davies KD and Earp HS: The TAM family: Phosphatidylserine sensing receptor tyrosine kinases gone awry in cancer. Nat Rev Cancer 14: 769-785, 2014.

4. Qu X, Liu J, Zhong X, Li X and Zhang Q: Role of AXL expression in non-small cell lung cancer. Oncol Lett 12: 5085-5091, 2016.

5. Zhang Z, Lee JC, Lin L, Olivas V, Au V, LaFramboise T, Abdel-Rahman M, Wang X, Levine AD, Rho JK, et al: Activation of the AXL kinase causes resistance to EGFR-targeted therapy in lung cancer. Nat Genet 44: 852-860, 2012.

6. Wilson C, Ye X, Pham T, Lin E, Chan S, McNamara E, Neve RM, Belmont L, Koeppen H, Yauch RL, et al: AXL inhibition sensitizes mesenchymal cancer cells to antimitotic drugs. Cancer Res 74: 5878-5890, 2014.

7. Byers LA, Diao L, Wang J, Saintigny P, Girard L, Peyton M, Shen L, Fan Y, Giri U, Tumula PK, et al: An epithelial-mesenchymal transition gene signature predicts resistance to EGFR and PI3K inhibitors and identifies Axl as a therapeutic target for overcoming EGFR inhibitor resistance. Clin Cancer Res 19: 279-290, 2013.

8. Wu F, Li J, Jang C, Wang $\mathrm{J}$ and Xiong $\mathrm{J}$ : The role of Axl in drug resistance and epithelial-to-mesenchymal transition of non-small cell lung carcinoma. Int J Clin Exp Pathol 7: 6653-6661, 2014.

9. Asiedu MK, Beauchamp-Perez FD, Ingle JN, Behrens MD, Radisky DC and Knutson KL: AXL induces epithelial-to-mesenchymal transition and regulates the function of breast cancer stem cells. Oncogene 33: 1316-1324, 2014. 
10. Gjerdrum C, Tiron C, Høiby T, Stefansson I, Haugen H, Sandal T, Collett K, Li S, McCormack E, Gjertsen BT, et al: Axl is an essential epithelial-to-mesenchymal transition-induced regulator of breast cancer metastasis and patient survival. Proc Natl Acad Sci USA 107: 1124-1129, 2010.

11. Mishra A, Wang J, Shiozawa Y, McGee S, Kim J, Jung Y, Joseph J, Berry JE, Havens A, Pienta KJ and Taichman RS: Hypoxia stabilizes GAS6/Axl signaling in metastatic prostate cancer. Mol Cancer Res 10: 703-712, 2012.

12. Antony J, Tan TZ, Kelly Z, Low J, Choolani M, Recchi C, Gabra H, Thiery JP and Huang RY: The GAS6-AXL signaling network is a mesenchymal (Mes) molecular subtype-specific therapeutic target for ovarian cancer. Sci Signal 9: ra97, 2016

13. Koorstra JB, Karikari CA, Feldmann G, Bisht S, Rojas PL, Offerhaus GJ, Alvarez H and Maitra A: The Axl receptor tyrosine kinase confers an adverse prognostic influence in pancreatic cancer and represents a new therapeutic target. Cancer Biol Ther 8: 618-626, 2009.

14. Kirane A, Ludwig KF, Sorrelle N, Haaland G, Sandal T, Ranaweera R, Toombs JE, Wang M, Dineen SP, Micklem D, et al: Warfarin blocks gas6-Mediated Axl activation required for pancreatic cancer epithelial plasticity and metastasis. Cancer Res 75: 3699-3705, 2015

15. Jemal A, Bray F, Center MM, Ferlay J, Ward E and Forman D: Global cancer statistics. CA Cancer J Clin 61: 69-90, 2011.

16. Chen W, Zheng R, Baade PD, Zhang S, Zeng H, Bray F, Jemal A, $\mathrm{Yu}$ XQ and He J: Cancer statistics in China, 2015. CA Cancer J Clin 66: 115-132, 2016.

17. Allum WH, Stenning SP, Bancewicz J, Clark PI and Langley RE: Long-term results of a randomized trial of surgery with or without preoperative chemotherapy in esophageal cancer. J Clin Oncol 27: 5062-5067, 2009.

18. Elkabets M,Pazarentzos E,Juric D, Sheng Q, Pelossof RA,Brook S, Benzaken AO, Rodon J, Morse N, Yan JJ, et al: AXL mediates resistance to PI3K $\alpha$ inhibition by activating the EGFR/PKC/mTOR axis in head and neck and esophageal squamous cell carcinomas. Cancer Cell 27: 533-546, 2015.

19. Paccez JD, Duncan K, Vava A, Correa RG, Libermann TA, Parker MI and Zerbini LF: Inactivation of GSK3 $\beta$ and activation of $\mathrm{NF}_{-} \mathrm{B}$ pathway via Axl represents an important mediator of tumorigenesis in esophageal squamous cell carcinoma. Mol Biol Cell 26: 821-831, 2015.

20. Hsieh MS, Yang PW, Wong LF and Lee JM: The AXL receptor tyrosine kinase is associated with adverse prognosis and distant metastasis in esophageal squamous cell carcinoma. Oncotarget 7 : 36956-36970, 2016.

21. Sawada G, Niida A, Uchi R, Hirata H, Shimamura T, Suzuki Y, Shiraishi Y, Chiba K, Imoto S, Takahashi Y, et al: Genomic landscape of esophageal squamous cell carcinoma in a japanese population. Gastroenterology 150: 1171-1182, 2016.
22. Su H, Hu N, Yang HH, Wang C, Takikita M, Wang QH, Giffen C, Clifford R, Hewitt SM, Shou JZ, et al: Global gene expression profiling and validation in esophageal squamous cell carcinoma and its association with clinical phenotypes. Clin Cancer Res 17: 2955-2966, 2011.

23. Michaylira CZ, Wong GS, Miller CG, Gutierrez CM, Nakagawa H, Hammond R, Klein-Szanto AJ, Lee JS, Kim SB, Herlyn M, et al: Periostin, a cell adhesion molecule, facilitates invasion in the tumor microenvironment and annotates a novel tumor-invasive signature in esophageal cancer. Cancer Res 70: 5281-5292, 2010

24. Subramanian A, Tamayo P, Mootha VK, Mukherjee S, Ebert BL, Gillette MA, Paulovich A, Pomeroy SL, Golub TR, Lander ES and Mesirov JP: Gene set enrichment analysis: A knowledge-based approach for interpreting genome-wide expression profiles. Proc Natl Acad Sci USA 102: 15545-15550, 2005.

25. Liberzon A, Birger C, Thorvaldsdóttir H, Ghandi M, Mesirov JP and Tamayo P: The molecular signatures database (MSigDB) hallmark gene set collection. Cell Syst 1: 417-425, 2015.

26. Xu J, Lamouille S and Derynck R: TGF-beta-induced epithelial to mesenchymal transition. Cell Res 19: 156-172, 2009.

27. Lamouille S, Xu J and Derynck R: Molecular mechanisms of epithelial-mesenchymal transition. Nat Rev Mol Cell Biol 15: 178-196, 2014.

28. Cui Y, Li YY, Li J, Zhang HY, Wang F, Bai X and Li SS: STAT3 regulates hypoxia-induced epithelial mesenchymal transition in oesophageal squamous cell cancer. Oncol Rep 36: 108-116, 2016.

29. Thiery JP, Acloque H, Huang RY and Nieto MA: Epithelial-mesenchymal transitions in development and disease. Cell 139: 871-890, 2009.

30. Singh A and Settleman J: EMT, cancer stem cells and drug resistance: An emerging axis of evil in the war on cancer. Oncogene 29: 4741-4751, 2010

31. Niwa Y, Yamada S, Koike M, Kanda M, Fujii T, Nakayama G, Sugimoto H, Nomoto S, Fujiwara M and Kodera Y: Epithelial to mesenchymal transition correlates with tumor budding and predicts prognosis in esophageal squamous cell carcinoma. J Surg Oncol 110: 764-769, 2014.

32. Bivona TG and Okimoto RA: AXL receptor tyrosine kinase as a therapeutic target in NSCLC. Lung Cancer (Auckl) 6: 27-34, 2015.

33. Sato K, Suda K, Shimizu S, Sakai K, Mizuuchi H, Tomizawa K, Takemoto T, Nishio K and Mitsudomi T: Clinical, pathological and molecular features of lung adenocarcinomas with AXL expression. PLoS One 11: e0154186, 2016.

34. Vogel C and Marcotte EM: Insights into the regulation of protein abundance from proteomic and transcriptomic analyses. Nat Rev Genet 13: 227-232, 2012. 